\title{
Inter y transdisciplinariedad en textos escolares de física: un estudio de dos casos
}

\author{
F. Javier Perales ${ }^{1}$ y Gonzalo Guerrero ${ }^{2}$ \\ ${ }^{1}$ Dpto. de Didáctica de las Ciencias Experimentales. Universidad de Granada (España). \\ ${ }^{2}$ Unidad de Innovación Educativa. Universidad de Santiago de Chile (Chile).
}

[Recibido el 1 de marzo de 2017, aceptado el 1 de junio de 2017]

La Física alberga múltiples relaciones con otras ciencias experimentales y humanas; no obstante, estas relaciones no suelen ser explicitadas en los libros de texto utilizados en la enseñanza obligatoria. Esta investigación analiza cómo se contempla la inter y transdisciplinariedad en libros de texto de Física. Para ello se evalúa, mediante una categorización derivada de un análisis de contenidos y de una revisión teórica, la consideración del enfoque Ciencia-Tecnología-Sociedad (CTS), la Historia y Naturaleza de la Ciencia, junto con la referencia hacia otras disciplinas. Se analiza un libro español y otro chileno de similar nivel educativo e importante difusión editorial. Los resultados evidencian una significativa presencia de los tópicos analizados pero con diferencias notables entre ambos textos. Como futuras líneas de investigación se plantean, entre otras, su extensión a libros de texto de otras disciplinas como Biología o Química.

Palabras clave: Libros de texto; Análisis de contenido; Física; Interdisciplinariedad; Transdisciplinariedad.

\section{Inter and Transdisciplinarity in Physics textbooks: A study of two cases}

Physics has multiple relationships with other experimental and human sciences; however, these relationships are not usually made explicit in textbooks used in compulsory education. This investigation analyze how inter and transdisciplinarity are considered in Physics textbooks. By means of a categorization derived from a content analysis and a theoretical revision is assessed how the Science-Technology-Society (STS), as well as the incorporation of the History and Nature of Science approaches and other subjects are presented. For it, two Spanish and Chilean textbooks from similar educational levels and brad editorial diffusion were reviewed. The results show a significant presence of the topics discussed but with relevant differences in both textbooks. Future lines of research should include, among others, its extension to textbooks of other disciplines such as Biology or Chemistry.

Keywords: Textbooks; Content analysis; Physics; Interdisciplinarity; Transdisciplinarity.

Para citar el artículo. Perales, F.J. y Guerrero, G. (2017). Inter y transdisciplinariedad en textos escolares de física: un estudio de dos casos. Ápice. Revista de Educación Científica, 1(1), 74-89. DOI: https://doi.org/10.17979/arec.2017.1.1.1992

Contacto.fperales@ugr.es,g.guerrero.fisica@gmail.com 


\section{Introducción}

La renovación de la enseñanza de la Física en los niveles educativos obligatorios pasa, entre otros factores, por acercarla a contextos cotidianos y próximos a los alumnos. No obstante, comúnmente los temas y contenidos de Física son tratados por los profesores en el aula mostrando una escasa relación con otras áreas de conocimiento o sin aplicaciones a la vida real, lo que conlleva una desmotivación o desinterés personal de los alumnos por este tipo de asignaturas. Sin duda, el alarmante descenso de las vocaciones científicas entre los jóvenes se debe, principalmente, a la forma en que se enseña la Ciencia en las aulas (Rocard et al., 2007).

El trabajo habitual con los contenidos disciplinares, ejemplos, ejercicios y problemas hace aparecer a la Física como una ciencia aislada, que no interactúa con otras áreas más allá de su necesaria relación con las Matemáticas. Sin embargo, la Física permite explicar la mayoría de los fenómenos naturales y aplicaciones tecnológicas, hallándose relacionada con disciplinas como la Biología, Química, Geología, Filosofía, Música, Arte, Filosofía, entre otras. De hecho, las últimas décadas han visto surgir disciplinas-frontera como la Biofísica, la Químico-Física o la Geofísica.

La realidad epistemológica e investigadora de la Física no suele explicitarse en los libros de texto de los niveles educativos no universitarios, sustrayendo por tanto a los estudiantes una de las señas de identidad de esta disciplina.

A pesar de que los textos actuales se rigen por marcos curriculares establecidos por órganos gubernamentales, dichas orientaciones muestran cierta flexibilidad al momento de abordarse desde las diferentes líneas editoriales, lo que permite incorporar la dimensión inter y transdisciplinar.

Una primera justificación de este trabajo puede hallarse en una perspectiva que trata de mejorar estos instrumentos de enseñanza sin ignorar el currículo implantado por cada país.

Una segunda justificación tiene que ver con la búsqueda de un cambio progresivo en el modelo didáctico de enseñanza de las ciencias, el cual debe tratar de evidenciar la inter y transdisciplinariedad, pero estar dirigido también a formar personas que sean ciudadanos responsables, involucrados y comprometidos en dar solución a problemas reales de la sociedad, actuando mas allá de un campo de conocimiento específico y evitando de esta forma una limitación de su acción. Los problemas globales actuales (cambio climático, energía, pobreza...) deben abordarse, hoy más que nunca, desde una perspectiva holística. Ello nos acercaría a la necesidad de potenciar una enseñanza de las Ciencias en contexto.

A pesar de las múltiples evidencias de la interconexión entre las diferentes disciplinas científicas, la traslación al ámbito curricular y, en concreto, al de los libros de texto, no ha sido objeto de investigaciones previas, a pesar de su indudable influencia en las ideas y actitudes de los alumnos frente a las Ciencias.

El problema de estudio se centra en indagar acerca de las referencias inter y transdisciplinares que se manifiestan en sendos libros de texto, tanto en España como en Chile. 


\section{Objetivos de la investigación}

1. Evaluar la inter y transdisciplinariedad presentes en textos escolares de Física mediante una categorización derivada de un análisis de contenidos y de una revisión teórica.

2. Analizar cualitativa y cuantitativamente cada una de las categorías de análisis basadas en la inter y transdisciplinariedad presentes en los textos de estudio, a fin de conocer su uso, misión y calidad.

3. Contrastar la inter y transdisciplinariedad presente en textos de Física de España y Chile.

\section{Marco teórico}

\section{Interdisciplinariedad, Multidisciplinariedad y Transdisciplinariedad en Ciencia}

Los conceptos que pretenden guiar nuestro análisis han sido objeto de interés desde distintas ópticas pero escasamente en el ámbito de la enseñanza. Seguramente ello está relacionado con la misma polisemia y complejidad de la definición de los términos implicados, tales como el de disciplina, interdisciplinariedad, multi o pluridisciplinariedad y transdisciplinariedad (Palmade, 1979), lo que ha conllevado propuestas confusas en el momento de promover intervenciones o plantear reformas al tratamiento de los programas de enseñanza de las ciencias.

Comenzaremos por mostrar el alcance que daremos en este trabajo a la palabra disciplina. Esta se refiere a un conjunto de conocimientos o habilidades que pueden enseñarse y aprenderse, siendo este el uso que aparece tempranamente ya en el siglo $\mathrm{V}$ en "De Septem Disciplinis" de Marciano Capella. Así, la "multidisciplinariedad", del latín "muchos", se refiere a una actividad asociada con muchas, múltiples o más de una disciplina existente, basándose en el conocimiento de ellas, pero manteniendo sus límites en cada campo. En tercer lugar, la "interdisciplinariedad", con su prefijo en latín "inter", que significa "entre", se refiere a una actividad entre disciplinas existentes con un carácter recíproco, analizando, sintetizando y armonizando vínculos entre aquellas en un todo, coordinado y coherente. Finalmente el prefijo "trans", del latín "más allá", viene a definir la "transdisciplinariedad" como la transcendencia de las disciplinas, logrando una integración de las ciencias sociales y naturales en un contexto de humanidad, y transgrediendo cada uno de sus límites (Choi y Pak, 2006).

Las definiciones anteriormente aportadas implican, por un lado, que la multidisciplinariedad sea contemplada como una pseudo-interdisciplinariedad, esto es, con una visión de un aditivo yuxtapuesto de las disciplinas involucradas, careciendo de intercomunicación, y haciendo que las disciplinas permanezcan separadas. Por el contrario, un buen ejemplo de coordinación de diferentes disciplinas se halla en la interdisciplinariedad, en donde se integran, enlazan, enfocan y se hacen interactuar diferentes disciplinas. Ejemplos concretos de esta última son la antropología económica o la biogeografía (Alvargonzález, 2011). Por otro lado, la transdisciplinariedad, como bien indica su nombre, pretende avanzar sobre estas dos últimas, ser unificadora, transgresora y transformadora, constituir un enlace crítico, integrador y teórico, con el cual nos referiremos al conocimiento que va más allá de los límites disciplinarios tras un proceso que reúne disciplinas y recambian información (Kerne, 2005; Choi y Pak, 2006; Klein, 2010) . Para lograr el trabajo transdisciplinar se requiere la deconstrucción, que acepta que un objeto puede pertenecer a diferentes niveles de la realidad, con las 
contradicciones que esto conlleva, sus paradojas y conflictos internos (Klein, 2004). La transdisciplinariedad pretende una articulación holística de las disciplinas, del conocimiento y del mundo. Hay que dejar claro que la desunión de las disciplinas científicas constituye un atributo inherente, que la separación, el aislamiento y la fragmentación existen (como resultado de la especialización), pero lo que se busca es afianzar los diferentes niveles que actualmente se encuentran fracturados, desunidos. Se pretende por tanto lograr la unificación de disciplinas heterogéneas, abarcando todo lo que está, no solo en ellas, sino entre y más allá de ellas, analizando su desarrollo y luego, de forma circular, plantearse el objetivo de comprender el mundo actual para la creación de una nueva realidad.

\section{Interdisciplinariedad y transdisciplinariedad en el currículo}

En el caso que nos ocupa existe una cierta tradición de Ciencia integrada auspiciada por organismos internacionales y que generó en la segunda mitad del siglo pasado un relevante número de proyectos curriculares (Gutiérrez, Fernández y Fernández, 1979).

No obstante, también existe habitualmente una fragmentación excesiva de los contenidos, los cuales se presentan discontinuos, desactualizados, además de con una pre-ponderación obsesiva al tratamiento de las Ciencias Biológicas en relación a las demás disciplinas que conforman las Ciencias de la Naturaleza. Esto apunta a la necesidad de generar un cambio en la manera en que se desarrollan los contenidos, en cómo se trabajan las habilidades científicas y en cómo están aprendiendo los estudiantes; una forma de aprender que puede atenuar estas debilidades es a través de la inter y transdisciplinariedad. Este hecho es admitido por psicólogos y educadores a la hora de plantear la enseñanza de las Ciencias en Educación Infantil y en los primeros cursos de Educación Primaria pero, lamentablemente, se abandona con posterioridad en aras de un conocimiento más especializado, lo que juega en contra de la percepción por parte de los estudiantes de los fenómenos naturales, que no soportan esas fragmentaciones artificiales.

Lo que se busca en esta nueva visión curricular (que posteriormente veremos de qué manera se ha intentado abordar desde los textos escolares) es conocer cómo se persigue este objetivo epistemológico, de qué manera se trabaja la reunificación del saber y el logro de un cuadro conceptual global, con carácter multifacético, complejo, que tiene la necesidad de obtener un saber consonante con la creciente interpelación entre CTS (Álvarez, 2004). Ello comporta necesariamente otro enfoque en la propia formación del profesorado.

Actualmente, la noción de contexto se considera básica al momento de hablar de currículos, y no hay duda de que enseñar Ciencias a partir del análisis de situaciones o problemas reales es un nuevo reto para el profesorado (Whitelegg y Parry, 1999; Gilbert, Bulte y Pilot, 2010). Para estos últimos autores la principal finalidad de la educación debe estar centrada en la alfabetización científica y el enfoque CTS, que llega para potenciar este proceso haciendo que los estudiantes se conviertan en ciudadanos que puedan participar en procesos democráticos, en la toma de decisiones y promoviendo la resolución de problemas científico-tecnológicos en nuestra sociedad (Membiela y Padilla, 2005). Dicha visión es recogida también por el enfoque competencial del aprendizaje en el marco del proyecto de evaluación PISA, concretamente en el documento elaborado por una comisión de expertos en Didáctica de las Ciencias presidida por Rodger Bybee (OECD-PISA, 2006). En este mismo sentido, es necesario preguntarse cómo seremos capaces de enfrentar los problemas 
curriculares para ir más allá de un área específica restringida en su acción si no hemos trabajado la visión de las Ciencias de manera ínter y transdisciplinar. La enseñanza de las Ciencias se debe llevar a contextos del mundo real en búsqueda de un aprendizaje significativo, ya que las explicaciones científicas e ideas sobre la Ciencia son necesarias para que los estudiantes den sentido a su vida futura en un mundo dominado por la Ciencia (Chamizo e Izquierdo, 2005).

\section{Interdisciplinariedad y transdisciplinariedad en los libros de texto}

Actualmente los libros de texto, tanto en Chile como en España, constituyen un reflejo de sus respectivos currículos oficiales y, si bien están elaborados desde la óptica de las disciplinas, esto no evita que sean sensibles a las dos dimensiones que se tratan de evaluar en este trabajo. Es importante destacar que en cada uno de los programas de ambos países, el enfoque CTS y la Historia de la Ciencia (HC) no son tratados como otras disciplinas; el primero constituye un enfoque curricular y el segundo una disciplina frontera. De todas maneras abordaremos su análisis ya que intrínsecamente se trabaja en base a una visión inter y transdisciplinar de la Ciencia.

Los libros de texto representan herramientas mediadoras que traducen y concretan aquellos significados incluidos en el currículo prescrito por los organismos gubernamentales y lo hacen a través de una presentación didáctica (Martínez, 2002; Gimeno, 2005). En este sentido, poseen un gran impacto debido al uso que le dan los profesores en el proceso enseñanza-aprendizaje de sus estudiantes; algunos estudios registran que el $99 \%$ de los profesores utilizan los libros de texto en sus aulas, convirtiéndolos así en la principal fuente de información disponible para el alumno (Caixeta de Castro y De Souza, 2010). Dichos textos son utilizados para la planificación y preparación de las clases, apoyo para las actividades, complemento del conocimiento de los profesores, aprendizaje de los estudiantes y para realizar las llamadas "investigaciones" bibliográficas escolares (Neto y Fracalanz, 2003). El libro de texto cumple asimismo la función actual de no trabajar solo contenidos, sino también las competencias y habilidades necesarias, mostrando un sentido pluralista en su uso (Cassab y Martins, 2008).

Es entonces por este impacto que el rol de los textos escolares resulta necesario de investigar, y junto con esto, alumbrar de qué manera se abordan los contenidos y cuál es el "estado del arte" en el tratamiento de los contextos disciplinares.

Los textos escolares pueden incluir temas de otras áreas, pero solo se ciñen a aquellos que son de impacto social, como por ejemplo la clonación y el efecto invernadero (Calvo y Martín, 2005; Martínez, Gil y Osada, 2003). A su vez un tratamiento interdisciplinar suele presentarse mediante "cajas de texto" llamadas "para saber más", "ventana a la historia", "curiosidades", con lo que no se trabajan de manera transversal ni en los contenidos como tales. Lo mismo ocurre con la Naturaleza de la Ciencia, quedando limitada solo a identificar al "descubridor" o a presentar la Ciencia de manera individualista en donde los científicos trabajan aisladamente descubriendo verdades a través de experimentos y excluyendo así los debates en el seno de las comunidades científicas (Muñoz y Bertomeu, 2003; Van Eijck y Roth, 2008). Junto con esto se presenta la Ciencia como una acumulación de conocimiento, sin desarrollar una capacidad crítica y reflexiva de los estudiantes sobre cómo se producen los cambios, cómo se desarrolla la Ciencia en contextos históricos y sociales que forman parte de su construcción, enfocándose solo en el producto final y no en el proceso. En definitiva, se excluye la HC y los aspectos filosóficos y epistemológicos (Rodríguez y Níaz, 2004). 
El tratamiento de la inter y transdisciplinariedad debe darse no solo de manera decorativa en un texto escolar, debe ir más allá de una simple viñeta o caja que acompañe un cúmulo de información descontextualizada y sin sentido para los estudiantes. Un material de estas características debe ser un material potencialmente significativo, que sea relacionable (o incorporable) a la estructura cognitiva de los estudiantes, de manera no arbitraria ni literal. Con esto los alumnos, por su parte, podrán llegar a ver el conocimiento de una disciplina, como es la Física, como parte de una completa red de valores y actividades que afectan a su entorno y a la sociedad. Así, la enseñanza de la Física en el contexto de situaciones del mundo real evitaría dar a los alumnos la impresión de que la Ciencia no tiene que preocuparse de los problemas de la sociedad, que los científicos solo se dedican a inventar teorías y hacer descubrimientos sin que les interese si estos se usan o no a beneficio de aquella. La enseñanza inter y transdisciplinar en el contexto del mundo, de la cultura, la sociedad y la ciudadanía proporciona un valor real a la educación científica y motiva a los estudiantes a encontrar contextos cercanos, ya sea la música, el arte, la historia, la tecnología, etc., con lo que se favorece una disposición positiva hacia el aprendizaje.

\section{Metodología}

\section{Diseño metodológico}

En el elenco de los diseños metodológicos que podemos encontrarnos en la investigación educativa, este trabajo puede enmarcarse en un estudio descriptivocomparativo. En el primer caso atenderemos a la identificación de indicadores de inter y transdisciplinariedad a través de un análisis de contenidos basado en categorías, extraídas por un procedimiento inductivo-deductivo. En el segundo caso compararemos los libros analizados mediante los criterios de presencia de inter y transdisciplinariedad.

\section{Muestra y técnicas de recogida de datos}

La muestra de este estudio está constituida por dos textos escolares de Física, ampliamente difundidos y que corresponden a niveles académicos similares entre los países. En el momento de realizar la investigación se encontraban en uso en centros educativos tanto en España, como en Chile, respectivamente:

1. Física-Química de tercer curso de ESO, editado en 2011 por la Editorial Anaya.

2. Física 10 año Medio, editado en 2013 por la Editorial Santillana.

\section{Instrumento}

Para la recolección de datos se elaboró un instrumento que posee, por un lado, la finalidad de medir la frecuencia (para un posterior análisis cuantitativo) con que se hacen referencias inter y transdisciplinares a lo largo de los textos en estudio y, por otro, evaluar en base a indicadores la calidad, uso y misión de estas referencias (para un posterior análisis cualitativo).

El instrumento fue construido a partir de una revisión de textos de Física, junto con un análisis de contenidos y una revisión teórica del tema, lo cual generó las distintas secciones y categorías del mismo. Para lograr una mayor objetividad y precisión se hace necesario definir estas secciones y explicitar cada una de ellas:

i. Contenido: por un lado se introducen los temas a estudiar, junto con ello se presentan los objetivos acompañados usualmente con preguntas para el trabajo de las ideas previas. Por otro lado, el contenido se refiere al "texto duro", o 
contenido disciplinar incluido en los textos. A su vez, este contenido contempla los textos que acompañan las imágenes a modo de "pie de foto".

ii. Actividades: secciones que aparecen mencionadas explícitamente como tales y que incluyen el trabajo con procedimientos científicos tales como: explicar, justificar, representar, identificar, calcular, describir, argumentar, esquematizar, inferir, observar, comprobar, enunciar leyes o teorías, reflexionar, dibujar, investigar, analizar, explicar. Dichos procedimientos serán contemplados si están presentes también en el planteamiento de preguntas y problemas transversalmente a lo largo de las unidades, como también en evaluaciones de progreso, de capítulo, de unidad y finales, sin ser estas de carácter práctico o experimentales.

iii. Ejemplos y ejercicios resueltos: ejemplos para hacer referencia a hechos, textos o cláusulas que se citen para comprobar, ilustrar o autorizar una afirmación, doctrina u opinión en un apartado o cuadro, fuera del contenido. Por su parte los ejercicios resueltos son secciones en que el autor muestra de manera explícita el desarrollo de ciertas actividades, que requieren la aplicación de lo estudiado, mostrando la forma y/o los pasos a seguir para resolverlas.

iv. Imágenes o ilustraciones: representaciones de seres, objetos o fenómenos (Perales, 2006). Por otro lado se hablará de ilustraciones al referirnos a imágenes más específicas, con carácter exclusivamente gráfico, y que acompañan a los textos escritos con la intención de complementar la información que suministran (Perales, 2006).

v. Prácticas experimentales: actividades en donde los alumnos deban resolver problemas desde la práctica y desde la manipulación de instrumentos o ciertos materiales. También se consideran prácticas experimentales las prácticas de laboratorio y/o la formulación de diseños experimentales que incluyen la elaboración o comprobación de hipótesis, formulación de preguntas de investigación, conclusiones, entre otras (Izquierdo, Sanmartí y Espinet, 1997).

vi. Cuadros laterales o ventanas de ampliación: cápsulas flotantes que complementan la comprensión de los temas tratados; estas son comúnmente del tipo "necesitas saber", "sabías que...", "para leer", "conexión con...", "ventana a...", "conéctate con...", "física y...", entre otras. Dichos cuadros serán considerados como tales si acompañan el tratamiento de los temas localizándose fuera de los párrafos donde se trata el contenido.

vii. Otros: secciones que no se encuentren dentro de las mencionadas anteriormente. Estas pueden ser resúmenes, cuadros o mapas conceptuales de cierre, entre otras.

El instrumento cuenta con columnas indicadoras de la disciplina o enfoque inter/transdisciplinar que mencionan en los textos. La primera de ellas se obtuvo en base a una revisión de textos de Física, en donde se encontraron en primer lugar los enfoques de Historia y Naturaleza de la Ciencia, CTS y, junto con estos, las conexiones con otras disciplinas; las más comunes fueron: Matemáticas, Ciencias Biológicas y de la Salud, Música, Arte y Cultura, Deportes, Política, Sociología, TIC, Economía y Geología.

Para no solo contabilizar la frecuencia de veces que se hace mención a cada uno de estos enfoques o disciplinas se elaboró una segunda columna, en donde se jerarquizan el tipo de referencias que se hacen para conocer en mayor profundidad cómo son. 
Dichos sub-indicadores se generaron a partir de misma revisión de textos de Física, fundamentados y contrastados posteriormente por una revisión bibliográfica.

A continuación se desglosa la elaboración del instrumento por enfoques y disciplinas encontrados, junto con sus sub-indicadores, justificando también la importancia de los enfoques:

i. HC: este primer enfoque interdisciplinar cuatro sub-indicadores:

a. Se hace referencia a uno o más personajes sin ser contextualizado(s).

b. Se hace una referencia biográfica de un personaje

c. Se hace referencia a un personaje contextualizando históricamente el desarrollo de sus aportes.

d. Se hace una reflexión del proceso dinámico, filosófico y epistemológico del desarrollo de la HCy sus protagonistas.

ii. Enfoque CTS: este segundo enfoque, en el cual está presente intrínseca e explícitamente esta inserción interdisciplinar, junto con la contextualización a través del enfoque CTS, está compuesto por cuatro sub-indicadores:

a. Se hace referencia a aplicaciones tecnológicas de los temas tratados.

b. Se hace referencia a problemáticas ambientales y/o contaminación.

c. Se introducen y desarrollan los conceptos o temas con un contexto centrado en las aplicaciones de la Ciencia y las interacciones entre la Ciencia y la Sociedad y/o se plantean controversias socio-científicas.

d. Se promueve la reflexión ciudadana en la resolución de problemas científicotecnológicos en nuestra sociedad.

iii. Matemática: para esta primera disciplina, los sub-indicadores son:

a. Se utilizan ecuaciones matemáticas para explicar un fenómeno.

b. Se hace referencia a problemas donde se utilicen cálculos matemáticos en situaciones no contextualizadas.

c. Se hace uso de explicaciones matemáticas mostrando los significados físicos de las expresiones utilizadas.

d. Se utilizan modelos, métodos y representaciones matemáticas para relacionar variables mostrando siempre un significado físico contextualizado.

iv. Ciencias Biológicas y de la Salud: para esta segunda conexión inter y transdisciplinar, los sub-indicadores encontrados fueron:

a. Se hace referencia a temas biológicos y/o ejemplificación con animales, plantas y otros seres vivos.

b. Se hace referencia a enfermedades, aplicaciones médicas y/o tecnología médica.

c. Se hace referencia a cuidados de la salud y/o nutrición.

v. Música, Arte y Cultura: para saber si se establecen conexiones con estas tres áreas y disciplinas los sub-indicadores identificados fueron:

a. Se hace referencia a aplicaciones relacionadas con música, instrumentos, entre otros.

b. Se hace referencia a cultura, interculturalidad, costumbres, actividades o comportamientos de la actividad humana.

c. Se hace referencia a historia del arte, literatura, procesos artísticos o audiovisuales. 
vi. Deportes y ocio: los sub-indicadores para esta disciplina y contexto fueron:
a. Se hace referencia a deportes o actividades deportivas.
b. Se hace referencia a situaciones de esparcimiento o entretenimiento tales como cine, teatro, televisión.
c. Se hace referencia a situaciones cotidianas de la vida diaria u hobbies.

vii. Política, sociología y economía : si bien fueron escasas las referencias encontradas sobre estas disciplinas en estudio, se agruparon y categorizaron en los siguientes sub-indicadores:
a. Se hace referencia a contextos o ideologías políticas.
b. Se hace referencia a aplicaciones económicas de los temas.
c. Se hace referencia al contexto social en cómo se desarrolla la Ciencia.

viii. TIC : si bien no se trata de una disciplina como tal, dada la importancia actual en la Sociedad y su enfoque interdisciplinar (ya que son parte de la mayoría de sectores: Educación, Robótica, Administración Pública, Empresas, Salud, etc.) se incluyó un indicador sobre las referencias al uso de las TIC en los libros de texto.

ix. Geología: se agregó esta disciplina, dadas las diferencias curriculares que existen en España y Chile en torno a la Geología. Se hizo una revisión de las referencias relacionadas con minerales, rocas, actividad volcánica, entre otras.

x. Astronomía: finalmente, luego de una revisión de contenidos en el texto de Chile, se encontraron referencias a esta disciplina por lo que se agregó como indicador.

\section{Validación}

Se llevó a cabo mediante el juicio de expertos (Escobar y Cuervo, 2008; Cabero y Llorente, 2013). Para la validación del instrumento desarrollado se empleó el juicio de expertos solicitándoles una valoración sobre los ítems e indicadores que componen dicha herramienta, así como una valoración global del mismo. Se trata, pues, de una validación de contenido cuyo objetivo es analizar y valorar los descriptores, categorías e indicadores utilizados para así comprobar si los ítems seleccionados miden todas las referencias que se desean medir.

En la Tabla I se sintetizan los aspectos considerados en el proceso de validación del instrumento.

Tabla I. Proceso de validación del instrumento

\begin{tabular}{|l|l|l|}
\hline \multicolumn{2}{|c|}{ Objetivos de la validación } & \multicolumn{2}{|c|}{ Expertos } & \multicolumn{2}{c|}{ Modo de validación } \\
\hline Analizar y valorar las categorías e & Tres expertos que & Método individual \\
indicadores del instrumento para & cuentan con una & $\begin{array}{l}\text { mediante el que se } \\
\text { obtiene la información de }\end{array}$ \\
evaluar las referencias inter y & experiencia superior a & obtiene los de expertos \\
transdisciplinariedad en libros de texto & $\begin{array}{l}\text { cinco años en trabajo } \\
\text { cada uno de los editorial o como } \\
\text { de Física. }\end{array}$ & $\begin{array}{l}\text { sin que los mismos estén } \\
\text { en contacto. }\end{array}$ \\
$\begin{array}{l}\text { Comprobar si faltan o se repiten algunas } \\
\text { categorías o indicadores, o si el } \\
\text { instrumento presenta un sesgo a favor } \\
\text { de alguno de los ítems. }\end{array}$ & & \\
\hline
\end{tabular}

Fuente: adaptado de Robles y Rojas (2015).

Tras recibir los informes de los expertos, se procedió a redefinir cada una de las categorías. Con los resultados se realizó un análisis cualitativo para evaluar la capacidad discriminativa de cada ítem a partir de los comentarios hechos por aquellos. De acuerdo 
con ello, se pudo inferir que, con respecto a la validez de contenido, las aportaciones cualitativas de los jueces consideraron apropiado el instrumento al propósito para el que había sido construido.

En la fase final de validación, se procedió a realizar una triangulación en la aplicación del instrumento con el propósito de obtener objetividad en los resultados, y junto con ello, una legitimación del instrumento. Se sometió al juicio de expertos las referencias encontradas en tres unidades para obtener la convalidación de sus categorías y comprobar si las mismas se repetían, si faltaban otras o si el peso de la evaluación estaba sesgado a favor de una de ellas; dichos resultados arrojaron concretamente algunas discrepancias que fueron consensuadas y discutidas.

Finalmente podemos decir que la aplicación del instrumento y posteriores resultados mostraron una alta coincidencia y grado de fiabilidad.

\section{Resultados}

Cuantitativamente, los datos obtenidos en este estudio se examinaron mediante un análisis estadístico de frecuencias, utilizando el Software Microsoft Excel. Para ello, se ingresaron en plantillas las referencias encontradas, indicando una breve reseña y el número de página de dicha referencia. Esto se hizo individualmente para cada una de las unidades de los libros de cada texto, a fin de obtener una visión de cada una de ellas. Posteriormente se realizó un conteo de frecuencias absolutas, acumuladas y relativas para obtener los resultados de cada categoría e indicador, y así disponer de una visión global de los libros de texto.

\section{Resultados del texto español}

En el texto aludido fueron encontradas un total de 621 referencias. En primer lugar cabe destacar que muchas de ellas fueron clasificadas en más de un indicador (ello ocurrió para ambos textos), lo que aumentó la frecuencia absoluta. Esto último será comentado posteriormente en la sección de discusión de los resultados. El mayor número de referencias se concentraron en el enfoque CTS, con 290, seguido de Matemáticas e HC con 139 y 73, respectivamente; las otras disciplinas quedan más atrás. En la Figura 1 se muestran los porcentajes de cada una de las referencias según enfoques y disciplinas.

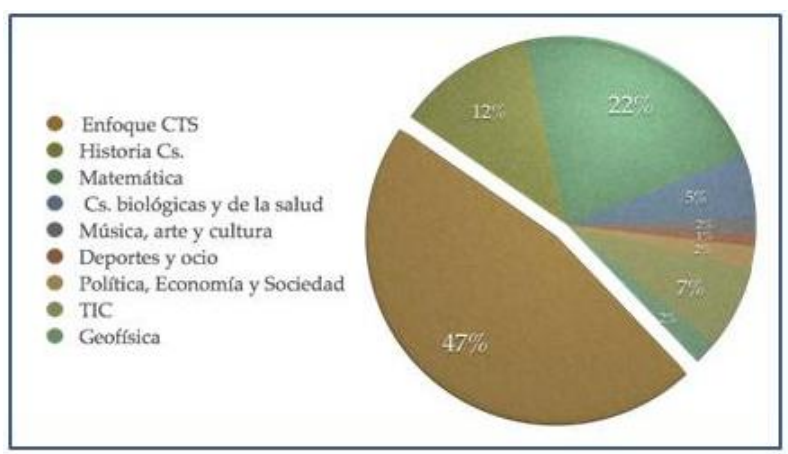

Figura 1. Porcentaje de referencias según enfoques y disciplinas en el texto español

Por otro lado, los resultados por secciones del texto muestran que las referencias encontradas se usan principalmente en las actividades del libro de texto, con 297 referencias, seguido de 124 referencias en el contenido disciplinar o "texto duro" del libro. Las referencias identificadas en imágenes fueron 94, seguida de 72 referencias en 
cuadros o ventanas laterales de ampliación. La Figura 2 muestra los porcentajes de las referencias encontradas para cada sección del libro de texto. Se observa claramente que, aproximadamente, la mitad de ellas se ubican en las actividades propuestas.

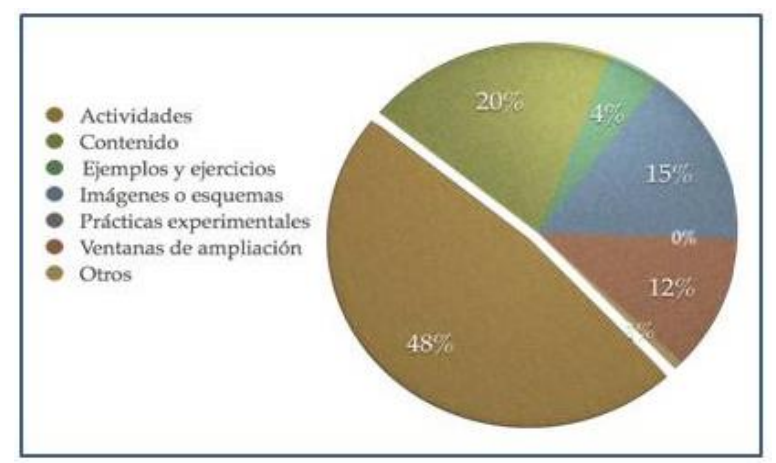

Figura 2. Porcentaje de referencias según secciones del texto español

\section{Resultados texto chileno}

En el texto de Chile fueron encontradas un total de 440 referencias. La mayoría de ellas fueron hacia la disciplina de Biología, con 143 referencias, seguidas del enfoque CTS, con 75 referencias; posteriormente las disciplinas de Música y Matemática con 67 y 59, respectivamente. En la Figura 3 se puede apreciar cómo casi un tercio de las referencias se enfocan hacia las Ciencias Biológicas, quedando con menor porcentaje, pero no por eso poco significativos, el enfoque CTS y Música.

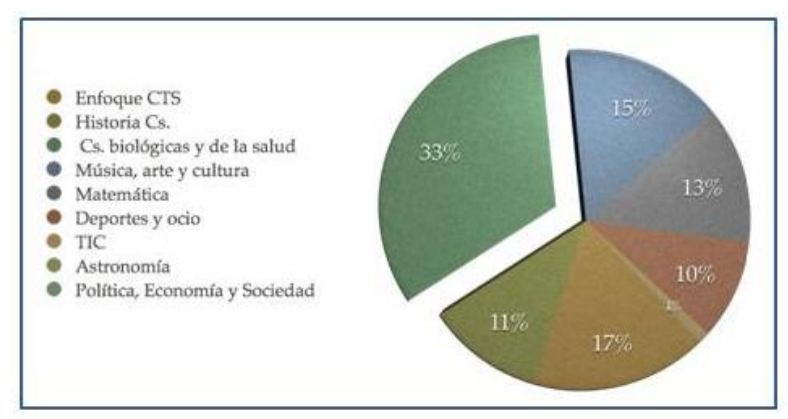

Figura 3. Porcentaje de referencias según enfoques y disciplinas en texto chileno

Los resultados arrojados del análisis estadístico muestran que la mayoría de las referencias inter y transdisciplinares se encuentran en las actividades, con 160 de las referencias, en segundo lugar en las imágenes, con 122. Finalmente en el contenido o "texto duro" del libro se hacen 107 reseñas. Lo anterior queda ilustrado de mejor forma en el siguiente gráfico (Figura 4), donde se observa claramente que más de un tercio de las referencias están en las actividades (36\%), seguido de un $28 \%$ de referencias en imágenes y un $24 \%$ en el contenido como tal. 


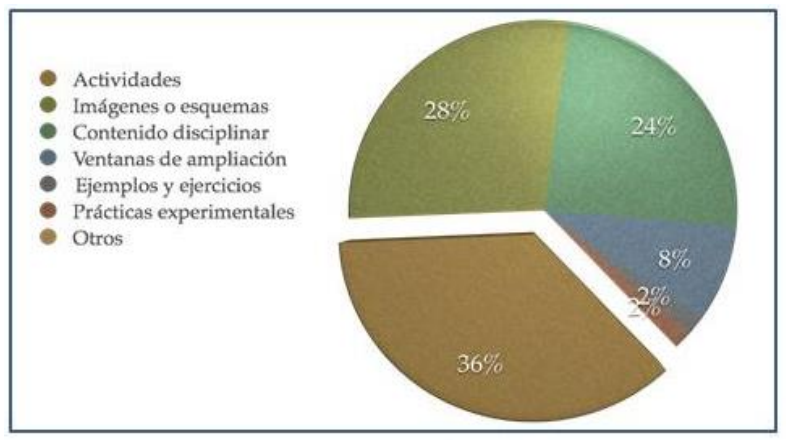

Figura 4. Porcentaje de referencias según secciones del texto chileno

\section{Discusión de los resultados}

Para el texto español, la mayoría de las referencias que hacen mención al enfoque HC se encuentran en la cuarta sub-categoría, en donde se hace un reflexión del proceso, dinámico, filosófico o epistemológico del desarrollo de la $\mathrm{HC}$ y sus protagonistas. Aunque esto demuestra un muy buen trabajo de este enfoque, solo está presente en algunas unidades y no de manera transversal en todo el texto.

Para el enfoque CTS, la mayoría de las referencias encontradas hacen mención a la primera y segunda sub-categoría, en donde se alude a aplicaciones o referencias hacia apartados tecnológicos, y en segundo lugar la mayoría de estas referencias lo son hacia problemáticas energéticas, ambientales y/o relacionadas con contaminación. De igual manera, la mayoría de estas referencias se concentraron solo en dos unidades mayoritariamente y no de manera holística en el texto, quedando unidades completas sin trabajar esta dimensión ni hacer referencias a otras disciplinas.

Es importante destacar también que para la disciplina de Matemáticas, las referencias encontradas hacen mención a problemas o actividades que involucran cálculos sin que sean contextualizadas a situaciones cotidianas, inter o transdisciplinares, y menos a contextos cercanos para los alumnos.

Finalmente, la mayoría de las referencias se encuentra en la sección "Actividades"; estas no son ejemplificadas con anterioridad, por lo que se le pide al alumno que aplique los contenidos vistos a problemas pero sin que se hayan contextualizado previamente con actividades demostrativas.

En el caso del texto chileno las referencias hacia la $\mathrm{HC}$ se concentran solo en dos de las cuatro unidades y aquellas no se encuentran en el contenido, sino mayoritariamente en las actividades o cuadros laterales, lo que muestra una diferencia con el texto español.

Por otro lado, el enfoque CTS se trabaja solo en la primera sub-categoría, en donde se hacen alusiones a aplicaciones tecnológicas o aparatos relacionados con tecnología, esto se muestra mayoritariamente en la primera y segunda unidad; básicamente se hace referencia en imágenes y se deja de lado el trabajo de involucrar a los alumnos en problemáticas ambientales o en la resolución de problemas científico-tecnológicos en la sociedad, es decir, sin implicarlos como ciudadanos.

Algo importante a destacar de este texto es el carácter interdisciplinar encontrado en las unidades de Sonido y Luz, en donde el trabajo con disciplinas como Biología, Medicina y Música está muy marcado, encontrando múltiples referencias en el contenido, en actividades e imágenes. 
Finalmente, en ambos textos se han hallado bastantes referencias inter $y$ transdisciplinares, mostrando que sí se pueden trabajar estas dimensiones en los libros de texto, independientemente del tema que se aborde. Algunos ejemplos de ello son descritos a continuación:

- En primer lugar, podemos observar cómo para el texto español los enfoques de HC y CTS se trabajan desde el comienzo de algunas unidades, de manera transversal, tanto en el contenido como en las actividades, mostrando una reflexión del proceso dinámico, filosófico y epistemológico del desarrollo de la $\mathrm{HC}$ y sus protagonistas.

- Para el texto chileno se encuentran referencias tanto inter como transdisciplinares, pero en algunas citas laterales llamadas "conexión con...".

- Finalmente se hallan referencias interdisciplinares, generalmente vinculadas con Biología y Medicina, como es la tendencia en este último texto, al final de cada unidad.

\section{Conclusiones}

Una de las limitaciones de este estudio fue el tamaño de la muestra, ya que hubiese sido conveniente contrastar un mayor número de ejemplares y textos de más países.

En líneas generales podemos mencionar que, si bien ambos libros de texto están elaborados desde una óptica disciplinar como es la Física, existen conexiones inter y transdisciplinares, aunque al mismo tiempo presentan grandes diferencias en cuanto a cómo se utilizan en cada uno de los textos. Por un lado, el texto español trabaja muy bien los enfoques de HC y CTS pero deja de lado la inter y transdisciplinariedad, quedando la mayoría de sus unidades sin conexiones con otras disciplinas, sin contextos ni aplicaciones a situaciones cercanas o cotidianas para los alumnos.

Por otro lado, el texto chileno resalta las conexiones, mostrando varias disciplinas que se ocupan simultáneamente de un idéntico problema, pero sin que exista entre ellas ninguna relación en cuanto a "cruzamientos" disciplinares, lo que nos lleva a hablar de interdisciplinariedad, pero no de transdisciplinariedad, ya que, si bien se estudian diferentes aspectos de unos problemas desde diferentes disciplinas mediante una agregación de competencias específicas de cada uno de ellas, no se pretenden borrar los límites que existen entre ellas para integrarlas en un ámbito único. Se siguen mencionando las "conexiones con..." en apartados, cuadros laterales o al final de las unidades del texto. También se observó que junto con posponer el trabajo inter y transdisciplinar para las últimas secciones o unidades en apartados, estas referencias no son abordadas de manera transversal en el texto.

El trabajo desde estas dimensiones es algo nuevo que se debe incorporar en los libros de texto, pero antes, y si bien se trata de un tema que se debe trabajar desde las líneas curriculares de los planes y programas de cada país, los autores, editores y adaptadores de los libros de texto disponen de libertad para mejorarlos a través de una adecuada selección de imágenes, creación de ejemplos, actividades, ejercicios y prácticas experimentales contextualizadas y transdisciplinares. En definitiva, es posible comenzar a trabajar desde este enfoque para futuros libros de texto, dejando de lado los libros centrados exclusivamente en contenidos disciplinares. Con ello se estará mostrando un libro de texto que evidencie una imagen de la Ciencia más próxima a la realidad, con problemas significativos, cercanos, motivadores y con múltiples referencias y aplicaciones a otras áreas del conocimiento u otras disciplinas, con lo que se ayudará a 
la construcción de contenidos, no solo conceptuales sino también basados en actitudes y aptitudes, junto con el trabajo de competencias y habilidades científicas.

A partir de la categorías enunciadas podemos mencionar que a través de la HC se debe mostrar la existencia de crisis en su evolución, cómo los conocimientos científicos, tanto en el ámbito general como de sus principales paradigmas, no son procesos lineales, ni inmutables sino procesos dinámicos en el tiempo, en donde participan colectivamente muchos científicos, con un constante intercambio de ideas inter y transdisciplinarmente. Ello conduce a que los alumnos reconozcan las implicaciones sociales de las principales aportaciones del pensamiento científico en el desarrollo general de la Humanidad y con ellas una visión más humanizada de la Ciencia.

En segundo lugar, el enfoque CTS debe estar presente transversalmente en los libros de texto, ya que no solo se deben construir conocimientos en base a contenidos disciplinares, sino que se deben trabajar dimensiones afectivas que la inter $y$ transdisciplinariedad pueden ayudar a desarrollar, poniendo énfasis en los valores y actitudes que favorezcan la participación ciudadana, y haciendo el cruce de disciplinas conectando otras áreas del conocimiento con aplicaciones de carácter multi y transdisciplinar. Se mostraría así la existencia de una implicación en cuestiones tecnológicas que afectan a procesos sociales $y$, junto con ello, derivaciones socioambientales.

Por otro lado, en Física las representaciones y modelos matemáticos producen un incremento de la comprensión de los contenidos, por lo que resulta de vital importancia que los alumnos puedan conocerlos y aplicarlos, para poder transformarlos y adaptarlos a su proceso de comprensión en múltiples áreas y contextos. Es por ello que dichas referencias deben estar en contextos para que adquieran un significado real y motiven a los estudiantes. La matemática como disciplina se mezcla con la Física con un carácter transdisciplinar, por lo que es necesario que este límite desaparezca y la matemática se convierta, en primera instancia, en un potente instrumento conceptual, para lo cual sus representaciones abstractas deben ser aplicables con sentido a nuevos problemas y, por qué no, a otras disciplinas.

Finalmente, la Biología, la Química, la Geofísica, la Astronomía y otras disciplinas científicas poseen tanto en común que se pueden utilizar bien inter o transdisciplinarmente en un libro de texto. Quedó demostrado en dos de las unidades del libro de texto de Chile cómo la Biología y la Música pueden fusionarse con la Física transdisciplinarmente, evidenciando que, como afirmara Pascal, "no se pueda entender el todo sin las partes, ni las partes con el todo". Creemos que queda patente en este trabajo que se pueden incluir dimensiones inter y transdisciplinares en un texto de Física, articulándolo de manera distinta, tomando visiones de otros países, líneas editoriales de otras áreas, junto con currículos que se adapten a estas dimensiones en lugar de agregar algunas de las disciplinas mencionadas, incluyendo el Arte, la Pintura, el Deporte y, por qué no, algunas tan controvertidas y casi sin referencias en estos dos textos como la Política y la Economía.

En cuanto a las líneas de continuidad de esta investigación, se considera adaptar el instrumento para ser aplicado a libros de texto de otras disciplinas como Biología o Química y saber de qué manera está presente la inter y transdisciplinariedad en otras áreas científicas. Otra de las líneas de continuidad sería emprender una revisión más profunda sobre el currículo de Física prescrito en ambos países, evaluando de qué manera se encuentran identificadas estas conexiones inter y transdisciplinares. $Y$, a su 
vez, otra dimensión de la investigación, algo más ambiciosa y a futuro, sería evaluar cómo está presente la inter y transdisciplinariedad en la formación inicial docente.

\section{Referencias bibliográficas}

Álvarez, M. (2004). La interdisciplinariedad en la enseñanza aprendizaje de las ciencias. En AA. VV. Didáctica de las ciencias: Nuevas perspectivas La Habana: Editorial Pueblo y Educación.

Alvargonzález, D. (2011). Multidisciplinarity, Interdisciplinarity,Transdisciplinarity, and the Sciences. International Studies in the Philosophy of Science, 25, 387-403.

Cabero, J. y Llorente, M. C. (2013). La aplicación del juicio de experto como técnica de evaluación de las tecnologías de la información (TIC). Eduweb. Revista de Tecnología de Información y Comunicación en Educación, 7(2), 11-22. Recuperado de: http://tecnologiaedu.us.es/tecnoedu/images/stories/jca107.pdf

Caixeta de Castro, M. E. y De Souza, P. (2010). Critérios que professores de química apontam como orientadores da escolha do livrodidático. Ensaio-Pesquisa Educação em Ciências, 12(2), 121-135.

Calvo, M. A. y Martín, M. (2005). Análisis de la adaptación de los libros de texto de ESO al currículo oficial, en el campo de la Química. Enseñanza de las Ciencias, 23(1), 17-32.

Cassab, M. y Martins, I. (2008). Significações de professores de ciências a respeito do livrodidático. Ensaio-Pesquisa Educação em Ciências, 10(1), 97-116.

Chamizo, J. A. e Izquierdo, M. (2005). Ciencia y contexto: una reflexión desde la filosofía. Alambique, 46, 9-17.

Choi, B. C. K., y Pak., A. W. P. (2006). Multidisciplinarity, interdisciplinarity and transdisciplinarity in health research, services, education and policy: 1. Definitions, objectives, and evidence of effectiveness. Clinical and Investigative Medicine, 29, 351-364.

Escobar, J. y Cuervo, A. (2008). Validez de contenido y juicio de expertos: una aproximación a su utilización. Avances en Medición, 6, 27-36. Recuperado de: http://www.humanas.unal.edu.co/psicometria/files/7113/8574/5708/Articulo3_J uicio_de_expertos_27-36.pdf

Gilbert, J., Bulte, A. y Pilot, A. (2010). Concept development and transfer in context based science education. Internacional Journal of Science Education, 1-21, First Article.

Gimeno, J. (2005). El currículum: ¿Los contenidos de la enseñanza o un análisis de la práctica? En: J. Gimeno Sacristán, y A. I. Pérez Gómez. Comprender y transformar la enseñanza. Madrid: Morata.

Gutiérrez, R., Fernández, J.M. y Fernández M. (1979). Bachillerato y Ciencia Integrada (I): Proyecto CIB. Madrid: Narcea.

Izquierdo, M., Sanmartí, N. y Espinet, M. (1997). Fundamentación y diseño de las prácticas escolares de ciencias experimentales. Enseñanza de las Ciencias, 17 (1), 45-59.

Kerne, A. (2005). Doing interface ecology: The practice of metadisciplinary. En ACM 
SIGGRAPH 2005 Electronic art and animation catalog. New York: Association for Computing Machinery.

Klein, J. T. (2004). Prospects for transdisciplinarity. Futures, 36, 515-526.

Klein, J. T. (2010). A taxonomy of interdisciplinarity. En R. Frodeman, J. T. Klein y C. Mitcham (eds.). The Oxford Handbook of Interdisciplinarity Oxford: Oxford University Press.

Martínez, J. (2002). Políticas del libro de texto escolar. Madrid: Morata.

Martínez, M., Gil, M. y Osada, J. (2003). Genetic engineering: a matter that requires further refinement in Spanish secondary school textbooks. International Journal of Science Education, 25(9), 1148-1168.

Membiela, P. y Padilla, Y. (Eds.) (2005). Retos y perspectivas de la enseñanza de las ciencias desde el enfoque Ciencia-Tecnología-Sociedad en los inicios del siglo XXI. Vigo: Educación Editora.

Muñoz, R. y Bertomeu, J. R. (2003). La historia de la ciencia en los libros de texto: la(s) hipótesis de Avogadro. Enseñanza de las Ciencias, 21(1), 147-159.

Neto, J. M. y Fracalanz, A. H. (2003). O libro didático de ciências: problemas e soluções. Ciência \& Educação, 9(2), 147-157.

OECD-PISA (2006). Marco conceptual para la evaluación PISA 2006. Paris: OECD Pub. Service.

Palmade, G. (1979). Interdisciplinariedad e ideologías. Madrid: Narcea.

Rocard, M. y otros (2007). Science Education now: A renewed pedagogy for the future of Europe. Bélgica: European Communities.

Perales, F. J. (2006). Uso (y abuso) de la imagen en la enseñanza de las ciencias. Enseñanza de las Ciencias, 24(1), 13-30.

Robles, P. y Rojas, M. D. C. (2015). La validación por juicio de expertos: dos investigaciones cualitativas en Lingüística aplicada. Revista Nebrija de Lingüística Aplicada, 9(12), 3-8.

Rodríguez, M. A. y Níaz, M. (2004). La teoría cinético molecular de los gases en libros de física: un perspectiva basada en la historia y filosofía de la ciencia. Journal of Science Education, 5(2), 68-72.

Van Eijck, M. y Roth, W. (2008). Representations of scientists in Canadian high school and college textbooks. Journal of Research in Science Teaching, 45(9), 1059-1082.

Whitelegg, E. y Parry, M. (1999). Real-life contexts for learning physics: meanings, issues and practice. Physics Education, 34(2), 68-72. 